Original
article

\title{
Risk factors for laparoscopically confirmed pelvic inflammatory disease: findings from Mumbai (Bombay), India
}

\author{
A Gogate, L Brabin, S Nicholas, S Gogate, T Gaonkar, A Naidu, A Divekar, A Karande, \\ C A Hart
}

Objectives: Sexually transmitted diseases (STDs) are an important cause of pelvic inflammatory disease (PID) but have often not been detected in microbiological studies of Indian women admitted to hospital gynaecology wards or private clinics. In this cross sectional study, women living in the inner city of Mumbai (Bombay) were investigated for socioeconomic, clinical, and microbiological risk factors for PID.

Methods: Microbiological tests and laparoscopic examination were carried out on 2736 women aged $\leqslant 35$ years who came to a health facility with suspected acute salpingitis or infertility or for laparoscopic sterilisation. 86 women with a clinical diagnosis of PID were not referred for laparoscopy although their characteristics are described. Associations between various risk factors and PID status were investigated and logistic regression performed on all factors that remained significant.

Results: Of women with a laparoscopically confirmed evaluation, 26 women had acute and 48 chronic pelvic infection. Independent risk factors for PID were later age at menarche $(\geqslant 14$ years), a history of stillbirth and no previous pregnancy, history of tuberculosis, STD, dilatation and curettage or previous laparoscopy, and presence of Gardnerella vaginalis.

Conclusions: It is concluded that STD related risk factors applied to only a small proportion of PID cases and that other determinants of PID are important, including obstetric complications, invasive surgical procedures such as laparoscopy, and tuberculosis.

(Sex Transm Inf 1998;74:426-432)

Keywords: laparoscopy; pelvic inflammatory disease; India

\section{Introduction}

It is difficult to assess how many women suffer from pelvic inflammatory disease (PID) in India or what the main causes of this disease are. Most information comes from gynaecological admissions to hospital but the number of cases so detected largely reflects the degree of awareness of personnel and the diagnostic criteria used. ${ }^{1}$ In sub-Saharan Africa sexually transmitted diseases (STDs) are the main cause of PID, ${ }^{2}$ but as far back as 1980 , the view was expressed by Muir and Belsey that tuberculosis, puerperal sepsis, and postabortal infections were relatively more important causes of PID in India. ${ }^{3}$ Earlier studies in Indian populations failed to detect gonococcal PID, ${ }^{4}$ and there have been relatively few published studies of Chlamydia trachomatis in relation to PID. ${ }^{5}$ In contrast, one study from a private clinic in Mumbai isolated genital mycoplasmas from $27.5 \%$ of PID cases, $15.7 \%$ of infertile women, and $3.7 \%$ of women undergoing tubal sterilisation. ${ }^{6}$

From 1993 to 1995 a study was undertaken in two inner city municipal hospitals in Mumbai to examine the socioeconomic, clinical, and microbiological characteristics of women admitted with suspected acute salpingitis or complaining of infertility. For comparative purposes, healthy, fertile women attending for laparoscopic sterilisation in a nearby postpartum centre were also routinely screened. The study sought to investigate risk factors for PID including prevalence of gonorrhoea, chlamydia, mycoplasmas, and bacterial vaginosis in women with and without PID as confirmed by laparoscopy. This paper describes the main determinants for PID that were found. HIV seroprevalence in the study population is reported elsewhere. ${ }^{7}$

\section{Methods}

STUDY POPULATION

A cross sectional study was conducted in Mumbai between October 1993 and December 1995, in association with the Brihan Mumbai Municipal Corporation. Study women were recruited at three centres-Lokmanya Tilak Municipal General Hospital, Mahim Maternity Home, and F-South Post Partum Centre. The women were recruited if they presented for gynaecological investigation, either with symptoms suggestive of acute pelvic infection or a history of infertility which might indicate previous salpingitis. Infertility was defined as inability to conceive during at least 12-18 months of cohabitation without any use of natural or modern contraception. Infertility cases related to a male factor were identified and excluded. In addition, fertile women seeking laparoscopic tubal ligation who had no symptoms of gynaecological disease were recruited as this provided an opportunity to screen a large group of asymptomatic women by laparoscopy. As acute salpingitis patients 
were likely to be younger than those seeking tubal ligation, women older than 35 years of age were excluded. Informed consent was obtained from all women, and procedures were carefully explained by auxiliary nurse midwives who had been specially trained using participatory methods.

RISK FACTORS

At recruitment, clinical and social questionnaires were administered. From questionnaires information was collected on variables hypothesised to be associated with PID. The clinical questionnaire covered reasons for referralgynaecological history including menstruation, symptoms of genital and urinary tract infections, obstetric, medical, and surgical histories. Subjects covered by the social questionnaire included sociodemographic information (family, marital status, religion, education, income); contraceptive use; and sexual history of the woman and her partner.

\section{GYNAECOLOGICAL EXAMINATION}

Consultants were brought together to agree on study procedures and overall supervision was provided by the consultant at Mahim (SG). The vulva was examined for lesions and vaginal discharge was noted. The cervix was inspected for ulcers, warts, ectopy, and cervicitis defined as erythema or inflammation with or without a mucopurulent discharge. Three high vaginal swabs were taken: one was placed in TV medium for Trichomonas vaginalis and candida; a second was placed in Amies transport medium for aerobes, anaerobes, and Gardnerella vaginalis, and a third was placed in sterile saline solution for wet mount and Gram staining. After the ectocervix had been cleaned of secretions, two Dacron coated swabs were placed in the cervical os and rotated to collect endocervical secretions and cells. These were placed separately in Amies transport medium for Gram stain and culture of Neisseria gonorrhoeae and culture of Mycoplasma hominis and Ureaplasma urealyticum. A third wire swab for antigen detection of Chlamydia trachomatis by ELISA was collected in Mastazyme transport medium. After removal of the speculum, a bimanual examination was carried out to test for rebound tenderness and masses, which were recorded diagrammatically, as well as tenderness of the fornices and excitation of the cervix. A clinical diagnosis of PID was made when lower abdominal pain was present, together with tenderness of the fornices, adnexal tenderness, or fever. After physical examination and before any operative procedure, a $5 \mathrm{ml}$ blood sample was taken for serological tests. At $\mathrm{F} / \mathrm{S}$ postpartum clinic, where only control cases were enrolled, transvaginal sonography was performed before all laparoscopic tubal ligations were done to rule out any pelvic pathology and to confirm pregnancy in cases of medical termination of pregnancy with tubal ligation.

\section{LAPAROSCOPY}

Before laparoscopy, routine haematological tests (erythrocyte sedimentation rate, white blood count, haemoglobin) were carried out. Laparoscopic diagnosis was carried out under general anaesthesia in the usual manner. Acute salpingitis was classified as follows: mild, if the tubes were inflamed but mobile, with no pus observed; moderate, if tubes were immobile and pus was present; severe, if pyosalpinx or an abscess was present. The presence of adhesions around the uterus and adnexae, with or without formation of tubal ovarian masses, or hydrosalpinx without any evidence of inflammation or exudate, was considered to be chronic pelvic infection. Before any further endoscopic procedure, $20 \mathrm{ml}$ sterile saline was inoculated into the cul de sac, sprayed over the uterus and adnexal areas and was aspirated back from the cul de sac. If any fluid was already in the cul de sac it was aspirated directly and returned to the laboratory for microbiological tests. Although obstetricians were requested to perform laparoscopy for all cases where acute salpingitis was suspected, most were extremely reluctant to perform laparoscopy for the more severe cases, as it was standard practice to treat such cases immediately on clinical grounds without performing laparoscopy. For these, culdocentesis was performed although it was recognised that cul de sac specimens obtained by this procedure would be open to interpretation. ${ }^{8}$ Women with acute salpingitis were started immediately on antibiotic therapy.

\section{MICROBIOLOGICAL ASSESSMENTS}

Samples were transported to the laboratory at Sion Hospital within 4 hours of collection.

\section{Microscopy}

Wet mount microscopy of high vaginal swabs was done immediately on arrival at the laboratory for $T$ vaginalis. Gram stained vaginal smears were examined microscopically for clue cells and white blood cells. Where $>5$ clue cells per high power field $(\times 40)$ were counted and white vaginal discharge had been clinically observed, a diagnosis of bacterial vaginosis was made. Gram stained cervical smears were also used for observation of diplococci, candida, and any other micro-organisms.

\section{Cultures}

Aerobes were inoculated into glucose phosphate broth and anaerobes into sodium thioglycollate broth. Gonococcal culture specimens were incubated at $35-37^{\circ} \mathrm{C}$ in carbon dioxide $(10 \%)$ for up to 48 hours on ThayerMartin medium. Sugar utilisation test was done on all oxidase positive Gram negative diplococci. $G$ vaginalis was grown on $\mathrm{GV}$ medium in $95 \%$ hydrogen and 5\% carbon dioxide. $T$ vaginalis specimens were cultured in AC medium. Sabouraud's agar was used for culture of candida. PPLO broth was used for ureaplasma and mycoplasma, followed by PPLO agar for colonies of $M$ hominis. All the media were prepared, inoculated, and incubated as routine microbiology procedures. 
Table 1 Proportion of women with genital infections and associated history, signs, and symptoms according to PID classification

\begin{tabular}{|c|c|c|c|c|}
\hline \multirow[b]{2}{*}{ Infection } & \multicolumn{3}{|c|}{ Laparoscopically confirmed } & \multirow[b]{2}{*}{ Clinically diagnosed $(n=86)$} \\
\hline & Acute $(n=26)$ & Chronic $(n=48)$ & Without PID $(n=2662)$ & \\
\hline$T$ vaginalis & $3.9(1)$ & $2.1(1)$ & $0.8(22)$ & $0.0(0)$ \\
\hline$G$ vaginalis & $19.2(5)$ & $12.5(6)$ & $7.4(196 / 2659)^{\star}$ & $15.1(13)$ \\
\hline Bacterial vaginosis & $0.0(0)$ & $2.1(1)$ & $0.5(13)$ & $8.1(7)$ \\
\hline Symptomatic candidosis & $0.0(0)$ & $0.0(0)$ & $0.0(0)$ & $5.8(5)$ \\
\hline N gonorrhoeae & $0.0(0)$ & $2.1(1)$ & $0.1(3 / 2659)$ & $0.0(0)$ \\
\hline$C$ trachomatis & $0.0(0 / 24)$ & $0.0(0 / 46)$ & $0.2(4 / 2599)$ & $1.2(1 / 85)$ \\
\hline$M$ hominis & $7.7(2)$ & $6.3(3)$ & $5.8(155 / 2655)$ & $5.8(5)$ \\
\hline U urealyticum & $11.5(3)$ & $20.8(10)$ & $21.2(565 / 2649)$ & $12.8(11)$ \\
\hline Reported vaginal discharge & $30.8(8)$ & $12.5(6)$ & $8.7(233) \dagger$ & $61.6(53) \ddagger$ \\
\hline History of STD & $3.8(1)$ & $2.1(1 / 47)$ & $0.1(3 / 2645) \dagger$ & $8.3(7 / 84)$ \\
\hline Partner infection & $7.7(2)$ & $6.2(3)$ & $4.8(125 / 2615)$ & $16.1(13 / 81)$ \\
\hline Cervicitis & $7.7(2)$ & $6.2(3)$ & $1.9(51)+$ & $16.3(14)$ \\
\hline
\end{tabular}

Numbers in parentheses used to calculate the percentage in each group unless otherwise stated.

* Significant difference $(\mathrm{p}<0.05)$ between laparoscopically confirmed PID cases and women with no PID

†Significant difference $(\mathrm{p}<0.01)$ between laparoscopically confirmed PID cases and women with no PID.

$\ddagger$ Significant difference $(\mathrm{p}<0.01)$ between clinically diagnosed and laparoscopically confirmed PID cases.

Antigen detection and serology

Cervical swabs of all cases as well as controls for chlamydia antigen were frozen at $-40^{\circ} \mathrm{C}$ until processed by an ELISA method (Mastazyme; Mast Laboratories, Bootle). Antigen detection of $C$ trachomatis by ELISA was performed on all the cases (suspected PID and infertility) and on every fifth specimen of the aspirates collected. All ELISA positive samples and a subsample of negatives were frozen and returned to Liverpool for PCR testing. PCR amplification was done by using both primers for $C$ trachomatis major outer membrane protein (MOMP in-house assay) and for the $C$ trachomatis cryptic plasmid (Roche). Occasionally problems of false negative arise from PCR detection owing to the presence of inhibitory factors (either destroying target DNA or inhibiting taq polymerase). In order to assess this possibility, $10 \%$ of PCR negative samples were retested having added some $C$ trachomatis. In each case $C$ trachomatis was detected (that is, no inhibitory factors were detected). Those samples that were positive by ELISA and confirmed by PCR were classified as positive for $C$ trachomatis. Samples that were positive by ELISA and negative by PCR were classified as negative for $C$ trachomatis, and similarly all ELISA negatives. The prevalence as cited in the results section was derived using this classification.

STATISTICAL METHODS

Significance was assessed using the $\chi^{2}$ or Fisher's exact test. In order to allow simultaneously for the effects of several confounding variables, logistic regression was used. Included in the regression equations were terms for all risk factors that were significant in the univariate analysis $(\mathrm{p}<0.2)$. The significance of each factor in the model was assessed using the likelihood ratio test. All analysis was performed using the STATA software. ${ }^{9}$

Permission to conduct the study was granted by the municipal corporation's appropriate authorities and ethical approval was obtained from the ethics committee of Lokmanya Tilak Hospital and Medical School.

\section{Results}

In all, 2879 women were eligible for the study, of whom 2736 underwent a laparoscopic examination. Seventy four women were found to have pelvic infection-26 with acute salpin-

Table 2 Demographic characteristics (\% (n)) of women according to PID classification

\begin{tabular}{|c|c|c|c|c|}
\hline & \multicolumn{3}{|c|}{ Laparoscopically confirmed } & \multirow[b]{2}{*}{ Clinically diagnosed $(n=86)$} \\
\hline & Acute $(n=26)$ & Chronic $(n=48)$ & Without PID $(n=2662)$ & \\
\hline \multicolumn{5}{|l|}{ Age group: } \\
\hline$<25$ years & $27.0(7)$ & $31.2(15)$ & $19.9(530)$ & $31.4(27)$ \\
\hline $25-29$ years & $50.0(13)$ & $39.6(19)$ & $45.8(1220)$ & $33.7(29)$ \\
\hline $30-35$ years & $23.0(6)$ & $29.2(14)$ & $34.3(912)$ & $34.9(30)$ \\
\hline Currently married: & $100.0(26)$ & $95.9(46)$ & $99.7(2653)^{\star}$ & $96.5(83)$ \\
\hline \multicolumn{5}{|l|}{ Education: } \\
\hline$\leqslant 3$ years & $50.0(13)$ & $41.7(20)$ & $40.8(1086)$ & $39.5(34)$ \\
\hline $4-9$ years & $34.6(9)$ & $35.4(17)$ & $42.6(1133)$ & $40.7(35)$ \\
\hline$\geqslant 10$ years & $15.4(4)$ & $22.9(11)$ & $16.6(44)$ & $19.8(17)$ \\
\hline Not known & & & (2) & \\
\hline \multicolumn{5}{|c|}{ Household income (per month): } \\
\hline$\leqslant 1000$ rupees & $15.4(4)$ & $20.8(10)$ & $13.2(351)$ & $25.6(22)$ \\
\hline $1001-3000$ rupees & $46.1(12)$ & $62.5(30)$ & $66.4(1767)$ & $58.1(50)$ \\
\hline$>3000$ rupees & $38.5(10)$ & $16.7(8)$ & $20.4(544)$ & $16.3(14)$ \\
\hline \multicolumn{5}{|l|}{ Religion: } \\
\hline Hindu & $73.1(19)$ & $75.0(36)$ & $73.6(1959)$ & $75.6(65)$ \\
\hline Muslim & $19.2(5)$ & $12.5(6)$ & $12.3(328)$ & $10.5(9)$ \\
\hline Other & $7.7(2)$ & $12.5(6)$ & $14.1(375)$ & $13.9(12)$ \\
\hline \multicolumn{5}{|l|}{ Place of birth: } \\
\hline Mumbai & $34.6(9)$ & $31.2(15)$ & $41.4(1099)$ & $29.1(25)$ \\
\hline Elsewhere & $65.4(17)$ & $68.8(33)$ & $58.6(1588)$ & $70.9(61)$ \\
\hline Not known & & & (8) & \\
\hline
\end{tabular}

*Significant difference $(\mathrm{p}<0.01)$ between laparoscopically confirmed PID cases and cases with no PID. 
Table 3 Reproductive indices (\% (n)) according to PID classification

\begin{tabular}{|c|c|c|c|c|}
\hline & \multicolumn{3}{|c|}{ Laparoscopically confirmed } & \multirow[b]{2}{*}{ Clinically diagnosed $(n=86)$} \\
\hline & Acute $(n=26)$ & Chronic $(n=48)$ & Without PID $(n=2662)$ & \\
\hline \multicolumn{5}{|l|}{ Age of menarche } \\
\hline$\geqslant 14$ years & $58.3(14 / 24)$ & $66.0(31 / 47)$ & $49.8^{\star}(1312 / 2632)$ & $50.0(42 / 84)$ \\
\hline Age at first sex & & & & \\
\hline$<18$ years & $42.3(11)$ & $37.5(18)$ & $41.1(1092 / 2659)$ & $55.8(48)$ \\
\hline \multicolumn{5}{|c|}{ Age at first pregnancy } \\
\hline$<18$ years & $28.6(4 / 14)$ & $24.0(6 / 25)$ & $21.4(528 / 2467)$ & $32.5(25 / 77)$ \\
\hline Never pregnant & $46.1(12)$ & $45.6(21 / 46)$ & $6.7+(178 / 2645)$ & $9.4(8 / 85) \ddagger$ \\
\hline \multicolumn{5}{|l|}{ History of stillbirths } \\
\hline Yes & $11.5(3)$ & $4.2(2)$ & $2.2 \dagger(59)$ & $4.6(4)$ \\
\hline \multicolumn{5}{|c|}{ Termination of pregnancy } \\
\hline Yes & $3.8(1)$ & $8.3(4)$ & $12.2(325)$ & $11.6(10)$ \\
\hline \multicolumn{5}{|c|}{ Spontaneous abortion } \\
\hline Yes & $7.7(2)$ & $8.3(4)$ & $6.9(184)$ & $8.1(7)$ \\
\hline \multicolumn{5}{|c|}{ Ever used oral contraceptives } \\
\hline Yes & $7.7(2)$ & $8.3(4)$ & $10.7(285)$ & $2.3(2)$ \\
\hline $\begin{array}{l}\text { Ever used IUCD } \\
\text { Yes }\end{array}$ & $7.7(2)$ & $8.3(4)$ & $22.0 \dagger(586)$ & $16.3(14)$ \\
\hline \multicolumn{5}{|l|}{ Ever used condom } \\
\hline Yes & $3.8(1)$ & $12.5(6)$ & $16.4(437)$ & $14.0(12)$ \\
\hline
\end{tabular}

*Significant difference $(\mathrm{p}<0.05)$ between laparoscopically confirmed PID cases and women with no PID.

†Significant difference $(\mathrm{p}<0.01)$ between laparoscopically confirmed PID cases and women with no PID

$\ddagger$ Significant difference $(\mathrm{p}<0.01)$ between laparoscopically confirmed and clinically diagnosed cases.

gitis and 48 with indications of chronic infection. No evidence of PID was found in the remaining 2662. Eighty six women were not referred for laparoscopy, but were considered on clinical grounds to have acute salpingitis; $6.8 \%$ of confirmed cases reported recent use of antibiotics.

EVIDENCE OF STDS

Lower tract infection

The prevalence of lower tract infection in the different categories of women with pelvic infection is shown in Table 1 . The most common organisms detected in all women were $G$ vaginalis and $U$ urealyticum. $G$ vaginalis was the only organism detected significantly more frequently in laparoscopically confirmed PID cases compared with women with no PID ( $\mathrm{p}<0.05)$. $N$ gonorrhoeae was cultured for only four women, three of whom did not have PID. There were five women with $C$ trachomatis infections, of whom four did not have PID. Women with clinically diagnosed PID reported a much higher prevalence of vaginal discharge $(61.6 \%)$ than other women $(p<0.01)$. Confirmed PID cases were more likely to report a vaginal discharge, give a history of STD, and have clinical evidence of cervicitis than women without PID ( $\mathrm{p}<0.01)$.

Upper tract infection

Among women with no visual signs of PID at laparoscopy, $M$ hominis was recovered from $0.08 \%$ (2), U urealyticum from $1.2 \%$ (31), and anaerobes from $0.04 \%$ (1) of cul de sac aspirates. Among women with laparoscopically confirmed PID, none of these micro-organisms was detected; $2.3 \%$ (12) of samples collected by culdocentesis were positive for $U$ urealyticum.

UNIVARIATE ANALYSIS OF RISK FACTORS FOR PID The other main results of the univariate analysis are given in Tables $2-4$. The demographic characteristics of women with and without PID were similar (Table 2). Table 3 shows various reproductive indices according to PID classification. Mean age of menarche for the whole sample was 13.5 years (SD 1.1); 63.4\% (45) of laparoscopically confirmed PID cases began menarche $\geqslant 14$ years of age compared with $49.8 \%$ (1312) of women without PID ( $p=$ 0.02 ). Age at first sex and first pregnancy was not associated with PID. In terms of obstetric history, women with laparoscopically confirmed PID had experienced fewer pregnancies and more stillbirths $(p<0.01)$. Whereas $45.7 \%$ of confirmed cases had never been pregnant, this was true of only $9.4 \%$ of clinically diagnosed cases $(p<0.001)$. No differences between PID groups were found with respect to previous spontaneous abortion or medical termination of pregnancy. No association was found between use ever of oral contraceptives or condoms. Ever use of an intrauterine contraceptive device (IUCD) was negatively associated with PID. A history of tuberculosis was given by $16.4 \%$ (12/93) of confirmed PID cases compared with $1.6 \% \quad(42 / 2653)$ of

Table 4 History of surgicalinvasive procedures (\% (n)) according to PID classification

\begin{tabular}{lllll}
\hline & \multicolumn{2}{l}{ Laparoscopically confirmed } & \\
\cline { 2 - 4 } & \multicolumn{1}{c}{ Acute $(n=26)$} & Chronic $(n=48)$ & Without PID $(n=2662)$ & Clinically diagnosed $(n=86)$ \\
\hline Appendicectomy & $3.8(1)$ & $0.0(0)$ & $0.7(20)$ & $2.3(2)$ \\
Peritonitis & $0.0(0)$ & $0.0(0)$ & $0.0(0)$ & $1.2(1)$ \\
Cervical cauterisation & $0.0(0)$ & $0.0(0)$ & $0.1(4)$ & $0.0(0 / 84)$ \\
Hysterosalpinogram & $0.0(0)$ & $14.6(7)$ & $0.4^{\star}(12 / 2660)$ & $0.0(0)$ \\
Dilatation and curettage & $15.4(4)$ & $31.2(15)$ & $5.4^{\star}(143 / 2659)$ & $9.3(8)$ \\
Laparotomy & $3.8(1)$ & $8.3(4)$ & $1.8^{\star}(47 / 2659)$ & $4.6(4)$ \\
Laparoscopy & $15.4(4)$ & $25.0(12)$ & $1.8^{\star}(48)$ & $36.0(31)$
\end{tabular}

*Significant difference $(\mathrm{p}<0.01)$ between laparoscopically confirmed PID cases and women with no PID. 
Table 5 Multivariate analysis of risk factors for PID

\begin{tabular}{lclc}
\hline Variables & $\begin{array}{l}\text { Adjusted } \\
\text { odds ratios }\end{array}$ & $\begin{array}{l}\text { 95\% Confidence } \\
\text { interval }\end{array}$ & $\begin{array}{l}\text { p Value } \\
\text { from LRS }\end{array}$ \\
\hline Currently unmarried & 42.8 & $(4.1,446.9)$ & 0.03 \\
Age at menarche $(\geqslant 14$ years) & 1.8 & $(1.0,3.3)$ & 0.05 \\
Never pregnant & 11.2 & $(5.8,21.8)$ & $<0.01$ \\
Stillbirths: 1 or more & 9.7 & $(3.3,28.4)$ & $<0.01$ \\
Ever use IUCD & 0.5 & $(0.2,1.4)$ & 0.20 \\
History of STD & 19.4 & $(1.7,217.3)$ & 0.02 \\
Vaginal discharge & 2.1 & $(0.9,4.9)$ & 0.11 \\
G vaginalis & 2.3 & $(1.0,5.3)$ & 0.07 \\
Cervicitis & 0.8 & $(0.1,4.1)$ & 0.74 \\
$T$ vaginalis & 3.9 & $(0.4,35.3)$ & 0.30 \\
Ever had hysterosalpinogram & 1.2 & $(0.3,4.9)$ & 0.81 \\
Ever had dilatation and curettage & 2.6 & $(1.1,6.1)$ & 0.03 \\
Ever had laparotomy & 1.6 & $(0.3,8.4)$ & 0.58 \\
Ever had laparoscopy & 6.0 & $(2.3,15.3)$ & $<0.01$ \\
History of tuberculosis & 8.9 & $(3.5,22.9)$ & $<0.01$ \\
\end{tabular}

${ }^{\star} \mathrm{LRS}=$ likelihood ratio test. referred for laparoscopy, so it is unlikely that their exclusion from the risk factor analysis biased the results. It was considered more rigorous to include only laparoscopically confirmed cases in the risk analysis, even though this meant combining acute and chronic cases.

While STDs are associated with a much increased risk of PID,${ }^{11}$ in a population with a low prevalence of STDs other determinants must be considered. Gardnerella vaginalis - but not bacterial vaginosis with which $G$ vaginalis is usually associated-was detected in $14.9 \%$ of PID cases and remained independently associated with PID. Medical termination of pregnancy was not a determinant of PID risk, perhaps because routine antibiotics are given to prevent complications from underlying genital tract infections such as bacterial vaginosis. ${ }^{12} 13$ The same applies to IUCDs which were the most popular modern contraceptive used by women. IUCD use is no longer considered to be a major determinant of PID risk beyond the first 20 days after insertion ${ }^{14}$ and Daling et al ${ }^{15}$ found that the lowest risk of tubal infertility was associated with use of a copper IUCD. Antibiotic coverage at the time of IUCD insertion and a low prevalence of STDs are likely to have eliminated much of the potential risk entailed in their use by study women. $G$ vaginalis was also not found in association with gonorrhoea or chlamydia in spite of the fact that bacterial vaginosis is usually described as having a role as a facilitator or as an opportunistic infection interacting with these virulent cervical pathogens. ${ }^{16}{ }^{17}$ Thus, other interactions of $G$ vaginalis with PID may be surmised.

We were not able to culture $G$ vaginalis from the upper genital tract but this probably reflects the fact that cul de sac aspirations, which are most accessible in surveys such as ours in Mumbai, are not the optimal method for isolating micro-organisms from the pelvic cavity. ${ }^{8}$ However, one of the more likely pathways for $G$ vaginalis to ascend to the upper genital tract would be as a result of obstetric complications, such as amniotic fluid infection or infection of the chorioamnion. ${ }^{18}$ An obstetric mechanism is also suggested by the fact that a history of stillbirths was an independent risk factor for PID. Prenatal infection with $G$ vaginalis may contribute to risk of stillbirth or $G$ vaginalis may ascend to the upper genital tract following a difficult labour-another cause of stillbirth. In Mumbai, most women deliver in a health facility although some women still return to their home villages for delivery. In this study, we were unable to perform endometrial biopsies, but it is likely that some women with PID, either concurrently or previously, had endometritis. ${ }^{19}$ Postpartum endometritis is one of the most common obstetric infections and in a study in Seattle $G$ vaginalis was the most common isolate from blood and from the endometrium of women with postpartum fever and endometritis. ${ }^{20}$ The high prevalence and increased concentration of particularly virulent micro-organisms in bacterial vaginosis patients is considered to be operative in the pathogenesis of postpartum endometritis. Whether These infections were also rare among women with clinical symptoms of PID who were not 
$G$ vaginalis is sufficiently virulent to cause tubal pathology is unclear.

Another indication that risk factors for PID in Indian women are different from Europe and from sub-Saharan Africa is indicated by the fact that $25.7 \%$ of women with confirmed PID had previously undergone surgical procedures such as dilatation and curettage (Table 4) - a procedure on which women themselves often insist, believing it to be helpful in curing many gynaecological problems - and it is commonly carried out in private clinics: $21.6 \%$ had undergone a previous laparoscopy (including tubal ligation) and this compared with $1.8 \%$ for women who did not have PID. Tubal ligation is vigorously promoted in India ${ }^{21}$ and although laparoscopy is normally regarded as a very safe procedure, in India thousands of sterilisations are performed in mass clinics in rural areas, ${ }^{22}$ and access to health facilities is so poor that complications would be difficult to evaluate. Mehta sent out questionnaires following his sterilisations camps, although a large proportion of women would have been illiterate..$^{22}$ In the present study, almost $60 \%$ of women had migrated to Mumbai-most from rural areas where their tubal ligations may have been performed. Laparoscopic tubal ligations are also frequently performed following medical termination of pregnancy $(37.9 \%$ of ligations in this study) and following hospital delivery $(37.1 \%)$. It is generally accepted that mini-laparotomy is the recommended approach for postpartum sterilisation after vaginal delivery, for less developed as well as for developing countries. ${ }^{23}$ Only when the surgeon is very experienced and the procedure carried out in a well equipped hospital is it regarded as acceptable. Even then women with no complications during labour, such as premature rupture of the membranes, ${ }^{24}$ or delivery complications, should be accepted. In rural India laparoscopy is preferred because it is a much quicker procedure for mass sterilisation camps than mini-laparotomy. In such camps, exclusion criteria are less rigorously applied. Mehta $^{22}$ reported including some women with previous abdominal surgery as well as women having had a recent abortion or birth. If PID does occur more frequently in this setting, particularly following MTP or recent pregnancy (with or without tubal ligation), it may account for the different range of micro-organisms found in this study compared with previous studies.

The other determinant of PID which emerged from this analysis was a history of tuberculosis. Women with no evidence of sexually transmitted organisms, who were found to have tubal occlusion, may have been cases of previous genital tuberculosis. Tubercular salpingitis once accounted for a significant proportion of salpingitis throughout the world. ${ }^{1}$ It is now a rarity in most developed countries, and its importance in developing countries is hardly known as it is a difficult condition to detect. ${ }^{25}$ Pulmonary tuberculosis remains a major problem in India. This study recruited women who lived in a most crowded and polluted environment, in the shadow of the textile mills, where tuberculosis is common. ${ }^{26}$ Tuberculosis is, none the less, a preventable and treatable infection, although treatment follow up rates for women are often disappointing. ${ }^{27}$

In conclusion, this paper presents risk factors for PID in Mumbai which differ from those which are classically based on Western studies, where STDs are more prevalent. Among Indian women at high risk of contracting STDs, like commercial sex workers, classic risk profiles will still be most discriminatory. For many women admitted to gynaecological wards in Mumbai, however, assessment and treatment based on those profiles may lead to suboptimal management. Moreover, risk of PID could probably be reduced by providing more information and appropriate reproductive health services for women with less emphasis on invasive operations. Most operational procedures carry a degree of risk and should not be used without good reason. If women were better informed about their disadvantages, they might choose to avoid using them unnecessarily (for example, by opting for birth spacing contraceptives rather than sterilisation). This, in turn, might reduce the incidence of pelvic infection in Mumbai.

This research was carried out by the Brihan Mumbai Municipal Corporation and the Population and Reproductive Health Programme at Liverpool. The study was funded by the Overseas Development Administration of the UK (now the Departmen for International Development). However, the Department for International Development can accept no responsibility for any information provided or views expressed. Many people contributed to the success of this study and for all their help we are grateful. We wish specifically to thank Dr S Desmukh, Dean of Lokmanya Tilak Hospital for facilitating work, and all who contributed to data collection in India, especially the clinical team, tributed to data collection in India, especially the clinical team, Which included Drs PR Vaidya, VR Badhwar, VR Ambiye, VD Patkar, PK Shah, JS Rege. In Liverpool we wish to thank Dr W
Tong for PCR testing of chlamydia samples in his laboratory.

Tong for PCR testing of chlamydia samples in his laboratory.
Contributors: A Gogate, principal investigator; L Brabin, prinContributors: A Gogate, principal investigator; L Brabin, prin-
cipal investigator; S Nicholas, statistician and responsible for data analysis; S Gogate, clinical coordinator and obstetrician involved in data collection and preparation of the manuscript; $T$ Gaonkar, senior laboratory technician, trained in Liverpool and contributing to all aspects of the project; A Naidu, laboratory technologist and data manager; A Divekar, laboratory technologist; A Karande, principal investigator; CA Hart, adviser, quality control for microbiology.

1 Pearce JM. Pelvic Inflammatory disease. A sexually transmitted disease with potentially serious sequels that is often treated poorly. BMF 1990;300:1090-1.

2 Brunham RC, Cheang M, McMaster J, et al. Chlamydia trachomatis, infertility and population growth in sub-Saharan Africa. Sex Transm Dis 1993;20:168-73.

3 Muir DG, Belsey MA. Pelvic inflammatory disease and its consequences in the developing world. Am F Obstet Gynecol 1980;138:913-28.

4 Kochar M. Etiology of pelvic infection treated by the gynecologic service of the Kasturba Hospital, Delhi, India. $A m$ f Obstet Gynecol 1980:138:872-4.

5 Luthra UK, Mehta S, Bhargava NC, et al. Reproductive tract infections in India: the need for comprehensive reproductive health policy and programs. In: Germain $\mathrm{A}$, Holmes KK, Piot P, Wasserheit JN, eds. Reproductive tract infections. New York: Plenum, 1992.

6 Gogate A. Mycoplasmal pelvic inflammatory disease. Int $f$ Med Microbiol 1990;(Suppl)20:239-41.

7 Brabin L, Gogate A, Gogate S, et al. Reproductive tract infections, gynaecological morbidity and HIV seroprevalence among women in Mumbai, India. Bull World Health Organ 1998;76:277-87

8 Sweet RL, Draper DL, Schachter J, et al. Microbiology and pathogenesis of acute salpingitis as determined by
laparoscopy: what is the appropriate site to sample. $A m \mathcal{F}$ Obstet Gynecol 1980;138:985-9.

9 STATA 4. Stata Corporation, 702 University Drive East, College Station, TX 77840, USA.

10 Mayaud P, Grosskurth H, Changalucha J, et al. Risk assessment and other screening options for gonorrhoea and chlamydial infections in women attending rural Tanzanian antenatal clinics. Bull World Health Organ 1995;75:621-30. 
11 Bevan CD, Johal BJ, Mumtaz G, et al. Clinical, laparoscopic and microbiological findings in acute salpingitis: report o a United Kingdom cohort. Br F Obstet Gynaecol 1995;102 407-14

12 Blackwell AL, Thomas PD, Wareham K, et al. Health gain from screening for infection of the lower genital tract in women attending for the termination of pregnancy. Lancet 1993;342:206-10.

13 Penney GC. Prophylactic antibiotic therapy for abortion. In: Templeton A, ed. The prevention of pelvic infection. London: RCOG Press, 1996:211-22.

14 Farley TMM, Rosenberg MJ, Rowe PJ, et al. Intra-uterine devices and pelvic inflammatory disease: an international devices and pelvic inflammatory disea

15 Daling JR, Weiss NS, Metch BJ, et al. Primary tubal infertility in relation to IUD use. N Engl f Med 1985;312:937-41.

16 Soper DE, Brockwell NJ, Dalton HP, et al. Observations concerning the microbial etiology of acute salpingitis. $A m \mathcal{F}$ Obstet Gynecol 1994:170:1008-17.

17 Blackwell AL. Anaerobic (bacterial) vaginosis and its complications. In: Templeton A, ed. The prevention of pelvic infection. London: RCOG Press, 1996:78-91.

18 Eschenbach D. Bacterial vaginosis: an overview. (Monograph.) Illinois: Curatek, 1993.

19 Korn AP, Hessol N, Padian N, et al. Commonly used diagnostic criteria for pelvic inflammatory disease have poor sensitivity for plasma cell endometritis. Sex Transm Dis 1995;22:335-41.
20 Rosene K, Eschenbach D, Thompkins LS, et al. Polymicrobial early post partum endometritis with facultative and anaerobic bacteria, genital mycoplasmas and Chlamydia trachomatis: treatment with piperacillin or cefoxitin. $\mathcal{F}$ Infect Dis 1986;153:1028-37.

21 International Institute for Population Sciences. National Family Health Survey, India. 1992-93. Bombay: IIPS, 1995

22 Mehta PV. A total of 250,136 laparoscopic sterilisations by a single operator. Br f Obstet Gynaecol 1989;96:1024-34.

23 World Health Organisation. Mini-incision for post-partum sterilization of women: a multicentre, multinational prospective study. Contraception 1982;26:495-501.

24 I-Cheng C, Gates D, Thapa S. Performing tubal sterlization during women's post-partum hospitalization: a review of the United States and international experiences. Obstet Gynecol Surv 1992;47:71-9.

25 Agarwal J, Gupta JK. Female genital tuberculosis-a retrospective clinico-pathological study of 501 cases. Ind $\mathcal{F}$ Pathol Microbiol 1993;36:389-97.

26 Ramasubban R, Crook N. Mortality toll of cities. Energy patterns of disease in Bombay. Econ Polit Weekly 1985;XX: 999-1005.

27 Hudelson P. Gender differentials in tuberculosis: the role of socio-economic and cultural factors. Tuber Lung Dis 1996 77:391-400. 